Palavras chave:

Resistência biológica

Biodegradação

Durabilidade natural

Histórico:

Recebido 29/06/201 I

Aceito 18/1 1/2013

Keywords:

Biological resistance

Biodegradation

Natural durability

Correspondência: marceloxisto@hotmail.com
DOI:
Marcelo Xisto Ribeiro', Lina Bufalino², Lourival Marin Mendes³, Vania Aparecida de Sá3, Alexandre dos Santos ${ }^{3}$, Gustavo Henrique Denzin Tonoli ${ }^{3}$

\section{RESISTÊNCIA DAS MADEIRAS DE PINUS, CEDRO AUSTRALIANO E SEUS PRODUTOS DERIVADOS AO ATAQUE DE Cryptotermes brevis}

RESUMO: Neste trabalho, objetivou-se comparar a resistência das madeiras de cedro australiano (Toona ciliata) e pinus (Pinus sp.) e de painéis aglomerados produzidos com essas espécies ao ataque do cupim de madeira seca Cryptotermes brevis, bem como quantificar a mortalidade dos insetos. Foram colocados 30 pseudo-operários em placas de Petri de acrílico de 9,5 cm de diâmetro, contendo as amostras (I,5 x I,5 x 0,5 cm) e folhas de algodão posicionadas sobre as tampas perfuradas umedecidas diariamente com $5 \mathrm{ml}$ de água. As placas foram acondicionadas em BOD (Biological Oxygen Demand) a $24 \pm 5^{\circ} \mathrm{C}$ e escotofase de $24 \mathrm{~h}$. Uma amostra testemunha sem fonte de alimentação foi incluída no bioensaio. $O$ cálculo do índice de deterioração (ID) das amostras e a avaliação da mortalidade foram realizados após 60 dias. Os cupins da amostra testemunha apresentaram mortalidade maior em relação aos cupins dos demais tratamentos, os quais não se diferenciaram entre si. A madeira de Toona ciliata foi mais resistente ao ataque de Cryptotermes brevis em relação à madeira de Pinus sp. Os índices de deterioração médios foram de $1,74 \%$ e 6,62\% para essas madeiras respectivamente. $O$ índice de deterioração médio do painel produzido com $100 \%$ de Toona ciliata (ID= I,58\%) foi similar ao índice de deterioração do painel produzido por essa mesma espécie em mistura com Pinus sp. (ID = I,87\%).

\section{RESISTANCE OF PINE, AUSTRALIAN RED CEDAR WOODS AND THEIR DERIVATE PRODUCTS TO Cryptotermes brevis ATTACK}

ABSTRACT: The aim of this work was to compare the resistance of Australian red cedar (Toona ciliata) and pine (Pinus sp.) woods and particleboards made from these species to dry-wood termite Cryptotermes brevis attack, as much as to quantify the mortality of the insects. 30 termite pseudo-workers were put in each 9,5 cm-diameter Petri dishes containing the samples $(1,5 \times 1,5 \times 0,5 \mathrm{~cm})$ and cotton sheets positioned on the perforated covers, daily moisturized with $5 \mathrm{ml}$ of water. The dishes were maintained in BOD (Biological Oxygen Demand) at $24 \pm 5^{\circ} \mathrm{C}$ and $24 \mathrm{~h}$ of escotophase. A control sample without any feed source was included in the bioassay. The deterioration index (ID) and mortality of the insects were evaluated after 60 days. Termites from the control sample presented higher termite mortality than the other treatments, which did not differ among each other. Toona ciliata wood was more resistant than Pinus sp. wood to Cryptotermes brevis attack. The average deterioration indexes were $1.74 \%$ and $6.62 \%$ respectively for those woods. The average deterioration index of the panels made with $100 \%$ Toona ciliata $(I D=1.58 \%)$ was similar to the deterioration index of particleboards made with this specie mixed with Pinus sp. (ID= I.87\%). 


\section{INTRODUÇÃO}

A Toona ciliata M. Roem, conhecida popularmente como cedro australiano, é uma Angiospermae de hábito arbóreo pertencente à Família Botânica Meliaceae. Ocorre naturalmente desde a Índia e Malásia até o Norte da Austrália (HERWITZ et al., 1998; KEENAN et al., 1997; SOUZA et al., 2009; TRIANOSKI, 20I0). Essa espécie apresenta crescimento eficiente em regiões com altitude de 500 a 1.500 metros e com precipitação anual de 800 a I.800 mm. No Brasil, encontrou condições favoráveis ao seu desenvolvimento vegetativo e produção de madeira (MURAKAMI, 2008; SOUZA et al., 2009; TRIANOSKI, 20I0).

A implantação da cultura da Toona ciliata é economicamente viável e confere investimento rentável ao produtor por ser uma espécie de crescimento rápido, quando comparada às espécies nativas brasileiras exploradas para serraria (SANTOS et al., 2010). Sua principal vantagem em relação ao cedro brasileiro é a resistência a ataques de Hypsipyla grandella, praga que ataca a gema apical de meliáceas, fazendo com que o tronco da árvore fique bifurcado. A Toona ciliata apresenta alta toxicidade às lagartas dessa broca, quando estas se alimentam de suas folhas. Por esse motivo, recomenda-se seu uso em plantios consorciados com mogno para ação de controle biológico (ALMEIDA, 2005; SANTOS et al., 2010).

As principais finalidades do cultivo de Toona ciliata, no Brasil, são produção de madeira para serraria e laminação (SANTOS et al., 2010; SOUZA et al., 2009). É considerada uma madeira de boa qualidade para usos nobres como fabricação de móveis, acabamentos em construção civil, construção naval e esculturas, com grande aceitação comercial em todo o mundo (BYGRAVE; BYGRAVE, 2005; LORENZI, 2003; PINHEIRO et al., 2003).

A qualidade de painéis compensados produzidos com Toona ciliata foi avaliada e resultados promissores foram encontrados (ALBINO et al., 20I I). Entretanto, é importante considerar que práticas como laminação e desdobro de qualquer espécie florestal geram resíduos madeireiros em abundância. $O$ baixo rendimento de serrarias relatado nos últimos anos resulta em uma grande quantidade de resíduos e, consequentemente, ocasiona uma forte tendência à utilização desse material, além de madeiras de qualidade inferior, para a produção de painéis aglomerados (DACOSTA et al., 2005).

O estudo da resistência natural de diferentes espécies de madeira e seus produtos derivados à biodegradação por agentes xilófagos é de suma importância para seleção e adequação de seu uso, uma vez que a maioria dos trabalhos existentes na literatura sobre painéis aglomerados trata, principalmente, de propriedades físicas e mecânicas. Dentre as inúmeras pragas de importância econômica para produtos à base de madeira, os cupins de madeira seca são os que mais se destacam. $O$ controle químico desses insetos têm sido o método mais eficaz para evitar ataques, mas os efeitos desses produtos são alarmantes em função dos problemas que podem criar ao homem e ao meio ambiente. Dessa forma, estudos sobre resistência natural são muito importantes para evitar o uso desnecessário de inseticidas (VERMA et al., 2009).

Diversos pesquisadores publicaram trabalhos de resistência natural de produtos à base de madeira a espécies de cupins (KARTAL; GREEN III, 2003; MELO et al., 2010; SILVA et al., 20I0; TERZI et al., 2009), porém, estudos sobre a resistência natural de produtos à base de madeira de reflorestamento ao cupim Cryptotermes brevis são bastante escassos na literatura. Gonçalves e Oliveira (2006) destacam-se no estudo da resistência de seis espécies florestais a essa praga. A espécie Cryptotermes brevis é considerada uma das mais destrutivas do mundo e tem como principal característica a alta resistência a ambientes de baixa umidade, justificando, assim, seu alto poder destrutivo (EDWARDS; MILL, 1986; LEPAGE, 1986).

Assim, neste trabalho, objetivou-se comparar a resistência das madeiras de Toona ciliata e Pinus sp. e de painéis aglomerados produzidos com essas espécies ao cupim de madeira seca Cryptotermes brevis, bem como quantificar a mortalidade dos insetos.

\section{MATERIAL E MÉTODOS}

\section{Matéria-prima}

Foram utilizados resíduos do desdobro de toras (costaneiras) de madeira de Toona ciliata M. Roem var. australis (I 8 anos), procedentes do município de Marechal Floriano/ES e tábuas de madeira de Pinus sp. (18 anos), procedentes do plantio experimental no Campus da Universidade Federal de Lavras (Lavras/MG). A densidade básica das madeiras foi determinada de acordo com a norma NBR II942 (ASSOCIAÇÃO BRASILEIRA DE NORMAS TÉCNICAS - ABNT 2003), enquanto que as análises químicas de teor de extrativos totais e lignina foram conduzidas de acordo com os procedimentos descritos pelas normas M3/69 (ASSOCIAÇÃO BRASILEIRA TÉCNICA DE CELULOSE E PAPEL - ABTCP, 1974a) e M70/7I (ABTCP, I974b), respectivamente. 


\section{Produção dos painéis}

Os painéis foram produzidos com partículas do tipo sliver, obtidas por meio do processamento de tábuas (Pinus sp.) e costaneiras (Toona ciliata), obtidas por moinho martelo com uma peneira de $6,14 \mathrm{~mm}$. A secagem dessas partículas foi realizada a $105 \pm 2^{\circ} \mathrm{C}$, até atingir a umidade base seca de aproximadamente $3 \%$.

O adesivo utilizado foi ureia-formaldeído (viscosidade de 618,94 cP, teor de sólidos de $57 \%$ e $\mathrm{pH}$ de 7,5 ) na proporção de $9 \%$ (base massa seca das partículas), além de emulsão de parafina na proporção de I,5\% (base massa seca das partículas),ambos aplicados com o auxílio de uma encoladeira do tipo tambor rotatório, por meio de aspersão, com pistola pneumática.

Em seguida, a massa de partículas tomou às dimensões de $480 \mathrm{~mm}$ de largura $\times 480 \mathrm{~mm}$ de comprimento e por meio da pré-prensagem (até 2 toneladas) e da prensagem, à temperatura de $160^{\circ} \mathrm{C}$, pressão de $3,92 \mathrm{MPa}$ e tempo de 8 minutos, o painel foi consolidado, apresentando densidade aparente e espessura nominais de $0,70 \mathrm{~g} \cdot \mathrm{cm}^{-3}$ e $15 \mathrm{~mm}$, respectivamente.

Foram produzidos dois tipos de painéis aglomerados, que tiveram sua densidade aparente determinada por meio das diretrizes da norma ASTM D-3345 (AMERICAN SOCIETY FOR TESTING AND MATERIALS - ASTM, 1994), com duas composições distintas: $100 \%$ Toona ciliata e $50 \%$ de Toona ciliata em mistura com $50 \%$ de Pinus sp.

\section{Obtenção e preparo dos corpos de prova}

Para as madeiras sólidas, uma peça, de cada espécie, de dimensões $200 \times 90 \times 25 \mathrm{~mm}$ (provenientes da transformação de tábuas e costaneiras) foi selecionada ao acaso. Foi utilizada a madeira da região do alburno. No caso dos painéis aglomerados as amostras foram retiradas de uma região central.

No total, foram obtidos quatro corpos de prova de cada material de dimensões de $10 \times 10 \times 15 \mathrm{~mm}$. Posteriormente, os corpos de prova foram mantidos em sala de climatização a uma temperatura de $20 \pm \mathrm{I}^{\circ} \mathrm{C}$ e umidade de $65 \pm 3 \%$, até estabilização de sua massa.

\section{Ensaio de resistência ao cupim Cryptotermes brevis}

Os cupins da espécie Cryptotermes brevis foram coletados em um móvel antigo de madeira de Araucaria angustifolia, durante a sua reforma. Pedaços de madeira foram cuidadosamente colocados em balde de plástico e, posteriormente, cobertos com papel alumínio para a proteção dos insetos durante o transporte.

A metodologia foi adaptada do trabalho realizado por Santos et al. (20II), utilizada para o cupim de montículo Cornitermes cumulans, no qual 50 indivíduos foram mantidos em cada placa de Petri de acrílico de $950 \mathrm{~mm}$ de diâmetro, forradas com papel filtro e contendo algodão umedecido diariamente sobre as tampas perfuradas com 13 orifícios de $1,5 \mathrm{~mm}$, à temperatura de $21 \pm I^{\circ} \mathrm{C}$ até a mortalidade de todos os indivíduos. Os autores posicionaram dietas artificiais de ágar, nutrientes e bagaço de cana-de-açúcar no centro das placas para manutenção dos cupins.

As adaptações, neste trabalho, consistiram em mudança no número de cupins pseudo-operários colocados por placa para 30 (em função do menor tamanho das colônias de cupim da espécie em estudo), na fonte de alimentação, sendo as dietas artificiais substituídas por amostras de madeira e painéis de madeira, tempo de condução e temperatura de manutenção do experimento. Outra alteração foi a utilização de placas de Petri não forradas com papel filtro, para garantir que os cupins se alimentariam das madeiras e painéis. Em todas as tampas foram feitos 12 orifícios de, aproximadamente, $2 \mathrm{~mm}$ de diâmetro, e foram colocados 30 operários por placa. Para umedecimento diário das placas com $5 \mathrm{ml}$ de água, três folhas de algodão de embalagem do tipo bobina foram sobrepostas e cortadas em pedaços de $20 \times 20 \mathrm{~mm}$.

Foram utilizadas quatro placas de Petri por tratamento, correspondentes aos materiais e à testemunha. As placas foram acondicionadas em BOD (Biological Oxygen Demand) a $24 \pm 5{ }^{\circ} \mathrm{C}$ e escotofase de $24 \mathrm{~h}$. Dentro de cada placa foi posicionada uma amostra de madeira ou painel aglomerado. Uma testemunha sem fonte de alimentação foi incluída no bioensaio para verificar se os cupins de fato se alimentariam. Os cupins mortos foram retirados diariamente sem reposição, conforme a metodologia de Santos et al. (201I). As análises do índice de deterioração das amostras e mortalidade foram verificadas após 60 dias em todas as placas. $O$ índice de deterioração (ID) foi calculado a partir da diferença das massas das amostras de madeira e painéis, antes e após o ataque, pela equação I, sendo ID = Índice de deterioração (\%); ma = massa da amostra climatizada antes do ataque $(\mathrm{g})$ e $\mathrm{md}=$ massa da amostra climatizada depois do ataque $(\mathrm{g})$.

$$
\mathrm{ID} \%=\frac{\mathrm{ma}-\mathrm{md}}{\mathrm{ma}} \cdot 100
$$




\section{Plano experimental e análise estatística dos dados}

O delineamento experimental foi inteiramente ao acaso, composto de cinco tratamentos estruturados de acordo com as condições de exposição dos cupins e quatro repetições (Tabela I).

TABELA I Plano experimental.

TABLE I Experimental design.

\begin{tabular}{|c|c|c|c|}
\hline Tratamento & Matéria-prima & Produto & Amostra \\
\hline Testemunha & - & - & Ausente \\
\hline $\mathrm{M}^{\mathrm{PI}}$ & Pinus sp. & \multirow{2}{*}{ Madeira } & \multirow{4}{*}{ Presente } \\
\hline$M^{\mathrm{TC}}$ & T. ciliata & & \\
\hline $\mathrm{P}^{\mathrm{TC}}$ & I00\% T. ciliata & \multirow{2}{*}{$\begin{array}{c}\text { Painel } \\
\text { Aglomerado }\end{array}$} & \\
\hline $\mathrm{P}^{\mathrm{TC} / \mathrm{PI}}$ & $\begin{array}{c}50 \% \text { T. ciliata }+ \\
50 \% \text { Pinus sp. }\end{array}$ & & \\
\hline
\end{tabular}

M: madeira; P: painel aglomerado; ${ }^{\text {TC: }}$ T. ciliata; ${ }^{\text {Pl: Pinus sp.; }}{ }^{\text {TC/PI: }} 50 \%$ T. ciliata e $50 \%$ Pinus sp.

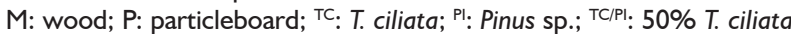
and $50 \%$ Pinus sp.

Os dados de mortalidade foram agrupados para a realização de contrastes de interesse, conforme apresentado na Tabela 2. As médias de índice de deterioração (ID) das madeiras de Toona ciliata e Pinus sp. e dos painéis com diferentes composições foram comparadas entre si por meio da análise de variância dos dados.

TABELA 2 Contrastes estatísticos realizados.

TABLE 2 Statistical contrasts carried out.

\begin{tabular}{ll}
\hline Especificação & Siglas \\
\hline I) Amostra Ausente $\times$ Amostra Presente & I) $A A \times A P$ \\
II) Madeira $\times$ Painel aglomerado & II) $M \times P$ \\
III) Pinus sp. $\times$ T. ciliata & III) $M^{\mathrm{PI}} \times M^{T C}$ \\
IV) $100 \%$ T. ciliata $\times 50 \%$ T. ciliata + & IV) $P^{T C} \times P^{T C / P I}$ \\
$50 \%$ Pinus sp. &
\end{tabular}

AA: amostra ausente; AP: amostra presente; M: madeira; P: paine

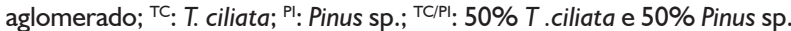
AA: absent sample; AP: present sample; M: wood; P: particleboard; TC:

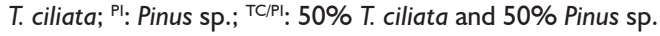

\section{RESULTADOS E DISCUSSÃO}

A caracterização química e física dos materiais de estudo está apresentada na Tabela 3. Os valores de densidade básica das madeiras são similares. Em relação à caracterização química, observa-se que o teor de extrativos totais da madeira de Toona ciliata foi superior à madeira de Pinus sp., enquanto que o teor de lignina da primeira foi inferior. Já, para painéis aglomerados, foi verificado que os valores de densidade aparente medidos foram similares ao valor pré-determinado (aproximadamente $0,7 \mathrm{~g} \cdot \mathrm{cm}^{-3}$ ). Apesar da semelhança entre os valores de densidade, ocorreu diferença de $10 \%$ entre os valores de razão de compactação.

TABELA 3 Caracterização básica dos produtos.

TABLE 3 Basic characterization of the products.

\begin{tabular}{ccccc}
\hline Produto & $\begin{array}{c}\text { Densidade } \\
\left(\mathrm{g} \cdot \mathrm{cm}^{-3}\right)\end{array}$ & $\begin{array}{c}\text { Razão de } \\
\text { Compactação }\end{array}$ & $\begin{array}{c}\text { Extrativos } \\
\text { Totais }(\%)\end{array}$ & $\begin{array}{c}\text { Lignina } \\
(\%)\end{array}$ \\
\hline $\mathrm{M}^{\mathrm{PI}}$ & $0,36^{\mathrm{b}}$ & - & 6,4 & 26,7 \\
$\mathrm{M}^{\mathrm{TC}}$ & $0,32^{\mathrm{b}}$ & - & 13,0 & 24,4 \\
$\mathrm{P}^{\mathrm{TC}}$ & $0,70^{\mathrm{a}}$ & 2,2 & - & - \\
$\mathrm{P}^{\mathrm{TC} / \mathrm{Pl}}$ & $0,69^{\mathrm{a}}$ & 2,0 & - & - \\
\hline
\end{tabular}

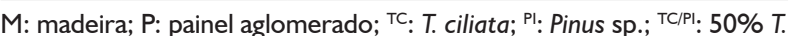
ciliata e $50 \%$ Pinus sp.

M: wood; P: particleboard; ${ }^{\text {TC: }}$ T. ciliata; ${ }^{\text {Pl: Pinus sp.; }}{ }^{\text {TC/PI: }} 50 \%$ T. ciliata and $50 \%$ Pinus sp.

Os valores médios de mortalidade dos cupins Cryptotermes brevis após 60 dias variou de $35 \%$ para o painel produzido com mistura de Toona ciliata e Pinus sp. a $83 \%$ para a testemunha (Figura I).

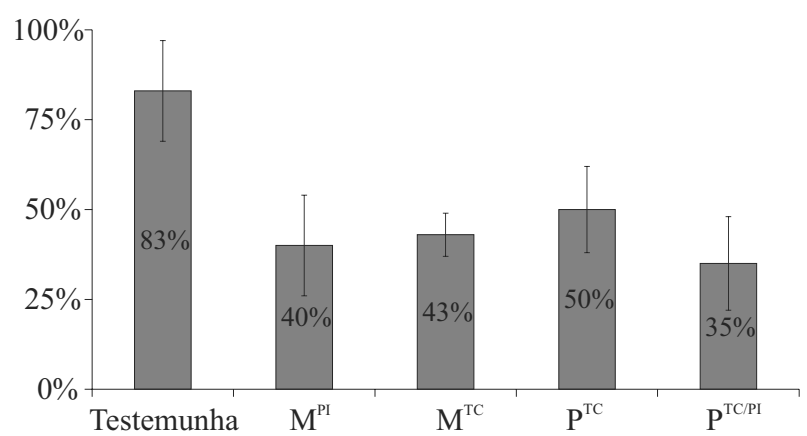

FIGURA I Mortalidade média acumulada de Cryptotermes brevis após 60 dias. ( $M$ : madeira; $P$ : painel aglomerado; ${ }^{\text {TC: }}$ T. ciliata; ${ }^{\text {PI: Pinus sp.; }}{ }^{\text {TC/PI: }}$ 50\% T. ciliata e $50 \%$ Pinus sp.)

FIGURE I Average accumulated mortality of Cryptotermes brevis after 60 days. (M: wood; P: particleboard; TC: T. ciliata; ${ }^{\text {Pl: }}$ Pinus sp.; TC/PI: $50 \%$ T. ciliata and $50 \%$ Pinus sp.)

Apenas o contraste realizado entre a testemunha e os demais tratamentos foi estatisticamente significativo (Tabela 4). É necessário esclarecer que na condução do experimento, a mudança no habitat e na alimentação disponibilizada para os insetos pode ter contribuído para a alta mortalidade da testemunha e à mortalidade parcial da população dos outros tratamentos. No entanto, pode- 
TABELA 4 Análise estatística dos dados de mortalidade TABLE 4 Statistical analysis of mortality data

\begin{tabular}{|c|c|c|c|}
\hline \multirow[b]{2}{*}{ Especificação } & \multirow{2}{*}{$\begin{array}{c}\text { Médias } \\
\text { contrastadas } \\
(\%)\end{array}$} & \multicolumn{2}{|l|}{ Parâmetros } \\
\hline & & F calculado ${ }_{\text {contraste }}$ & $\begin{array}{l}\hat{Y} / C \\
(\%)\end{array}$ \\
\hline I) Amostra A x AP & $83 \times 42$ & $27,962 *$ & 40 \\
\hline II) $M \times P$ & $42 \times 43$ & $0,014^{\mathrm{ns}}$ & - \\
\hline III) $M^{P I} \times M^{T C}$ & $40 \times 43$ & $0,116^{\mathrm{ns}}$ & - \\
\hline IV) $P^{T C} \times P^{T C / P I}$ & $50 \times 35$ & $2,349^{\text {ns }}$ & - \\
\hline \multicolumn{4}{|c|}{ F calculado experimental $=5,898^{\text {significativo }}$} \\
\hline \multicolumn{4}{|c|}{ Coeficiente de Variação = 25,37 \% } \\
\hline
\end{tabular}

AA: amostra ausente; AP: amostra presente; M: madeira; P: painel aglomerado; ${ }^{\text {TC: }}$ T. ciliata; ${ }^{\text {Pl: Pinus sp.; }}$ TC/PI: $50 \%$ T. ciliata e $50 \%$ Pinus sp.; $\hat{Y} / C$ : estimativa de contraste dividido pelo maior coeficiente de contraste

AA: absent sample; AP: present sample; M: wood; P: particleboard;

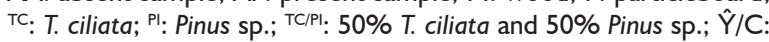
contrast estimative divided by the higher contrast coefficient

se dizer que a mortalidade observada na testemunha foi superior às observadas para os demais tratamentos em $40 \%$, indicando que os cupins se alimentaram das amostras lenhosas oferecidas, o que contribui para a diminuição da mortalidade final.

Não houve diferença estatística significativa no contraste realizado entre os painéis e as madeiras sólidas. Os painéis aglomerados contêm adesivo ureiaformaldeído e parafina, além de terem sido prensados sob alta temperatura e apresentarem maior densidade aparente. Dessa forma, nenhuma dessas propriedades teve influência sobre a mortalidade dos insetos.

Os contrastes entre as madeiras sólidas $\left(\mathrm{M}^{\mathrm{PI}} \mathrm{e}\right.$ $\left.M^{C E}\right)$ e entre painéis aglomerados $\left(P^{C E}\right.$ e $\left.P^{C E / P I}\right)$ não foram significativos. Dessa forma, a presença de substâncias potencialmente inseticidas na madeira de Toona ciliata não ocasionou alta mortalidade dos cupins, ao contrário do observado por Gonçalves e Oliveira (2006) para o cedro rosa (Cedrela fissilis). Para os painéis aglomerados foi possível verificar que as diferentes composições também não influenciaram na mortalidade dos cupins.

NaFigura2, estãoapresentadososvaloresmédios para índice de deterioração (ID) para os tratamentos contendo madeira e painéis aglomerados. Foram obtidos índices de deterioração (ID) estatisticamente diferentes para as madeiras ( $F$ calculado $=13,709 ; C V=43,98 \%$ ), sendo a madeira de Toona ciliata mais resistente em relação à madeira de Pinus sp. Esse resultado demonstra que, mesmo que altos teores de extrativos não causem a morte do agente xilófago, algumas substâncias presentes em tecidos vegetais podem exercer efeito repelente e inibir a alimentação do inseto (GALLO et al., 2002).

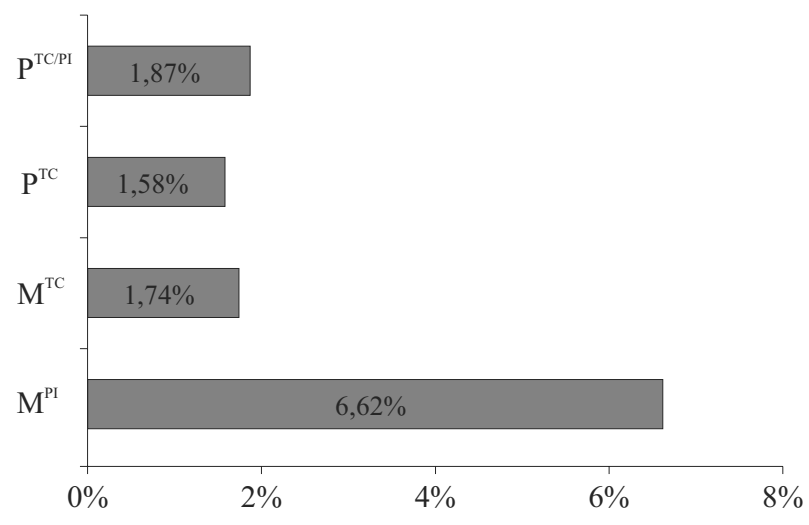

FIGURA 2 Valores médios do índice de deterioração para cada material. (M: madeira; P: painel aglomerado; TC: T. ciliata; ${ }^{\text {PI. }}$ Pinus sp.; ${ }^{\mathrm{TC} / \mathrm{PI}}$ : 50\% T. ciliata e $50 \%$ Pinus sp.)

FIGURE 2 Average values of deterioration index for each material. (M: wood; P: particleboard; TC: $T$. ciliata; ${ }^{\mathrm{PI}}$ : Pinus sp.; ${ }^{\mathrm{TC} / \mathrm{PI}}$ : 50\% T. ciliata and $50 \%$ Pinus sp.)

A madeira Toona ciliata apresenta menor densidade básica em relação à madeira de Pinus sp., fator que teoricamente facilitaria a deterioração e a penetração do inseto na amostra, o que não ocorreu. Esse resultado demonstra que o efeito químico pode ser o mais relevante para a resistência natural das madeiras. Gonçalves e Oliveira (2006) também verificaram que a madeira de cedro rosa (Cedrela fissilis) apresentou menor degradação por cupins em relação à madeira de pinus, quando amostras foram submetidas ao ataque de Cryptotermes brevis.

Os limonóides ou tetratriterpenóides são os componentes inseticidas mais extraídos de espécies da família Meliaceae. Essas substâncias também são conhecidas como meliacinas por seu sabor amargo que pode inibir a ingestão do alimento (ARAÚJO et al., 2009). Naliteraturaexistemevidências da presença de limonóides na madeira da espécie Toona ciliata. Foi verificada alta mortalidade de Heterotermes tenuis submetidos a extratos Cedrelona e I,2-Dihidroxicedrelona obtidos da madeira dessa espécie (SEVERINO et al., 2007). Em outro estudo realizado para controlar o aparecimento de pústulas de fungos, a cedrelona obtida da madeira de Toona ciliata, foi o componente mais eficiente (GOVINDACHARI et al., 2000).

As amostras do painel composto por 100\% de madeira de Toona ciliata apresentaram índice de deterioração estatisticamente igual aos painéis compostos por $50 \%$ dessa espécie e $50 \%$ de Pinus sp. $(\mathrm{F}$ calculado $=0,204 ; \mathrm{CV}=52,02 \%)$. Assim, a inclusão da madeira de Toona ciliata contribuiu para a proteção 
do aglomerado produzido com madeira de Pinus sp. a agentes xilófagos. Kartal e Green III (2003) afirmam que painéis aglomerados produzidos com madeiras com composição química que lhes confira maior resistência tendem a apresentar menor susceptibilidade a agentes xilófagos.

\section{CONCLUSÕES}

A mortalidade dos cupins da testemunha, sem a presença de fonte de alimentação, é superior à dos cupins alimentados com madeira e painéis de madeira, para os quais não houve diferença.

A madeira de Toona ciliata apresenta maior resistência (menor índice de deterioração - ID) ao ataque de Cryptotermes brevis em relação à madeira de Pinus sp. A substituição parcial (50\%) da madeira de Pinus sp. pela madeira de Toona ciliata na composição de painéis aglomerados resultou em ID similar aos painéis produzidos com $100 \%$ de Toona ciliata.

A alta resistência de Toona ciliata pode estar relacionada á presença de substâncias nocivas presentes nos extrativos de sua madeira, principalmente limonóides. Conduziu-se este trabalho, com o objetivo de contribuir para $\circ$ entendimento da resistência ao ataque de cupins de madeira seca em madeiras e produtos derivados da madeira, demonstrando a possibilidade de produzir painéis de madeira mais resistentes a cupins.

\section{REFERÊNCIAS}

ALBINO, V. C. S.; SÁ, V. A.; BUFALINO, L.; MENDES, L. M.; ALMEIDA, N. A. Avaliação das propriedades físicomecânicas de painéis compensados de Toona ciliata $M$. Roem. var. australis. Cerne, Lavras, v. I7, n. I, p. 103-108, jan./mar. $201 \mathrm{l}$.

ALMEIDA, G. B. Criação contínua de Hypsipyla grandella (Zeller, I848) (Lepidóptera: Pyralidae) com dieta artificial. 2005. 62 f. Dissertação (Mestrado em Agronomia) - Universidade Federal Rural da Amazônia, Manaus, 2005.

AMERICAN SOCIETY FOR TESTING AND MATERIALS. ASTM D-3345: standard method for laboratory evaluation of wood and other cellulosic materials for resistance to termite. Annual Book of ASTM Standard, Philadelphia, v. 4I0, p. 439-44I, 1994.

ARAUJO, S. A. C.; TEIXEIRA, M. F. S.; DANTAS, T. V. M.; MELO, V. S. P.; LIMA, F. E. S.; RICARTE, A. R. F.; COSTA, E. C.; MIRANDA, A. M. Usos potenciais de Melia azedarach L. (Meliaceae): um levantamento. Arquivos do Instituto Biológico, São Paulo, v. 76, n. I, p. |4 | - |48, jan./mar. 2009.
ASSOCIAÇÃO BRASILEIRA DE NORMAS TÉCNICAS. NBR I 1 942: madeira: determinação da densidade básica. Rio de Janeiro, 2003.

ASSOCIAÇÃO BRASILEIRA TÉCNICA DE CELULOSE E PAPEL. ABTCP-M3/69: métodos de ensaio. São Paulo, 1974a.

ASSOCIAÇÃO BRASILEIRA TÉCNICA DE CELULOSE E PAPEL. ABTCP-M70/7I: métodos de ensaio. São Paulo, 1974b.

BYGRAVE, F. L.; BYGRAVE, P. L. Growing Australian red cedar and other Meliaceae species in plantation. Canberra: RIRDC, $2005.60 \mathrm{p}$

DACOSTA, L. P. E.; HASELEIN, C. R.; SANTINI, E. J.; SCHNEIDER, P. R.; CALEGARI, L. Qualidade das chapas de partículas aglomeradas fabricadas com resíduos do processamento mecânico da madeira de Pinus elliottii Engelm. Ciência Florestal, Santa Maria, v. 15, n. 3, p. 3 II322, set. 2005.

EDWARDS, R.; MILL, A. E. Termites in buildings: their biology and control. Felcourt: Rentokil, 1986. 23I p.

GALLO, D.; NAKANO, O.; CARVALHO, R. P. L.; BAPTISTA, G. C.; PARRA, J. R. P.; ZUCCHI, R. A.; ALVES, S. B.; VENDRAMIM, J. D.; MARCHINI, L. C.; LOPES, J. R. S.; OSOMOTO, C. Entomologia agrícola. São Paulo: FEALQ/USP, 2002. 920 p.

GONÇALVES, F. G.; OLIVEIRA, J. T. S. Resistência ao ataque de cupim-de-madeira seca (Cryptotermes brevis) em seis espécies florestais. Cerne, Lavras, v. 12, n. I, p. 80-83, jan./ mar. 2006.

GOVINDACHARI, T. R.; SURESH, G.; GOPALAKRISHNAN, G.; MASILAMANI, S.; BANUMATHI, B. Antifungal activity of some tetratriterpenoids. Fitoterapia, Novara, v. 7I, n. 3, p. 317-320, jun. 2000.

HERWITZ, S. R.; SLYE, R. E.; TURTON, S. M. Redifining the ecological niche of a tropical rain forest canopy tree species using airborne imagery: long-term crown dynamics of Toona ciliata. Journal of Tropical Ecology, Cambridge, v. I4, n. 5, p. 683-703, Sept. 1998.

KARTAL, S. N.; GREEN III, F. Decay and termite resistance of medium density fiberboard (MDF) made from different wood species. International Biodeterioration \& Biodegradation, Birmingham, v. 5I, n. I, p. 29-35, Jan. 2003.

KEENAN, R.; LAMB, D.; WOLDRING, O.; IRVINE, T.; JENSEN, R. Restoration of plant diversity beneath tropical tree plantations in Northern Australia. Forest Ecology and Management, Amsterdam, v. 99, p. |17-131, May 1997. 
LEPAGE, E. S. Química da madeira. In: Manual de preservação de madeiras. São Paulo: IPT, 1986. p. 69-98.

LORENZI, H. Árvores exóticas no Brasil: madeireiras, ornamentais e aromáticas. Nova Odessa: Instituto Plantarum, 2003. 385 p.

MELO, R. R.; SANTINI, E. J.; HASELEIN, C. R.; GARLET, A.; PAES, J. B.; STANGERLIN, D. M. Resistência de painéis aglomerados produzidos com diferentes proporções de madeira e casca de arroz a fungos e cupins xilófagos. Ciência Florestal, Santa Maria, v. 20, n. 3, p. 50I-5II, jul./set. 2010.

MURAKAMI, C. H. C. Cedro australiano: valorização de espécies nobres. Informativo Florestal do Norte Pioneiro Florestal, Piracicaba, ano 2, n. 7, p. 2-4, fev. 2008.

PINHEIRO, A. L.; LANI, L. L.; COUTO, L. Cultura do cedro australiano para produção de madeira serrada. Viçosa, MG: UFV, 2003. 42 p.

SANTOS, A.; ZANETTI, R.; BUFALINO, L.; SILVA, W. L. P. Sobrevivência de operários do cupim-de-montículo Cornitermes cumulans Kollar, 1832 (ISOPTERA: TERMITIDAE) alimentados com diferentes dietas. Arquivos do Instituto Biológico, São Paulo, v. 78, p. | 5 | I54, jan./mar. 20II.

SEVERINO, R. P.; FERNANDES, J. B.; VIEIRA, P. C.; SILVA, M. F. G. F.; BATISTA PEREIRA, L. G.; CAMARGO-DIETRICH, C. G. C.; PEREIRA, D. A.; COSTA-LEONARDO, A. M.; BUENO, O. C. Biological activity of limonoids from Meliaceae against a subterranean termite (Heterotermes tenuis). Sociobiology, Chicago, v. 50, n. 3, p. 947-957, 2007.
SILVA, F. C.; TREVISAN, H.; SILVA, K. E. D.; LELIS, R. C. C.; CARVALHO, A. G. Influência de diferentes adesivos utilizados na fabricação de painéis aglomerados na ação de Coptotermes gestroi (Wasmann, 1986) (Isoptera: Rhinotermitidae). Floresta, Curitiba, v. 40, n. 2, p. 379384, ago. 2010.

SOUZA, J. C. A. V.; BARROSO, D. G.; CARNEIRO, J. G. A. Cedro Australiano (Toona ciliata). Niteroi: Rio Rural, 20I0. 12 p. (Manual Técnico, 2I).

SOUZA, J. C. A. V.; BARROSO, D. G.; CARNEIRO, J. G. A.; TEIXEIRA, S. L.; BALBINOT, E. Propagação vegetativa de cedro-australiano (Toona ciliata M. Roemer) por miniestaquia. Revista Árvore, Viçosa, v. 33, n. 2, p. 205213, mar./abr. 2009.

TERZI, E.; KÖSE, C.; BÜYÜKSARI, U.; AVCI, E.; AYRILMIŞ, N.; KARTAL, S. N. Evaluation of possible decay and termite resistance of particleboard containing waste tire rubber. International Biodeterioration \& Biodegradation, Birmingham, v. 63, n. 6, p. 806-809, Sept. 2009.

TRIANOSKI, R. Avaliação do potencial de espécies florestais alternativas, de rápido crescimento, para produção de painéis de madeira aglomerada. 2010. 262 p. Dissertação (Mestrado em Manejo Florestal) Universidade Federal do Paraná, Curitiba, 2010.

VERMA, M.; SHARMA, S.; PRASAD, R. Biological alternatives for termite control: a review. International Biodeterioration and Biodegradation, Birmingham, v. 63, n. 8, p. 959-972, Dec. 2009. 
